\title{
Improving junior doctor experience at Sheffield Teaching Hospitals
}

\author{
Authors: Daniel Gatt* and Jennifer Hill
}

\section{Introduction}

Sheffield Teaching Hospitals Foundation Trust (STHFT) is one of the largest UK foundation trusts with 17,000 staff, approximately 1,000 junior doctors (JDs) in training and around 700 career grade doctors. The previous chief executive officer and medical director had a vision to improve the relationship between junior doctors and senior management in the trust. By fostering this relationship, the ultimate goal was to make the JD cohort feel connected, involved and valued by the organisation which would make STHFT an attractive workplace and result in excellent clinical outcomes and a high level of patient satisfaction.

\section{Methods}

A number of listening events were hosted by the previous chief executive and medical director at the trust. These events were attended by JDs as well as clinical directors and senior leaders in the trust. While many ideas emerged from the discussions, four main themes were identified.

$>$ Connect JDs within the trust with people, systems and processes.

$>$ Create a great training experience.

$>$ Create a healthy workplace to work and thrive.

$>$ Engagement in improvement.

One of the deputy medical directors was established as lead for JDs experience in the trust, and the chief registrar role was firmly embedded in this initiative to gain a stronger representation of the JDs workforce, as well as becoming a JD champion.

A JDs Oversight Group now meets regularly and reports on its progress to the clinical management board, and the JDs Forum.

\section{Results}

The areas of work to date have included.

$>$ Improving medical handover.

> Producing induction videos for new JDs.

$>$ Developing quality standards for rota management.

$>$ Evening leadership connection events for JDs with invited speakers.

Authors: Sheffield Teaching Hospitals Foundation Trust ${ }^{*} \mathrm{RCP}$ chief registrar
> Engaging JDs in quality improvement.

> Developing a business case for the provision of new doctors' mess facilities.

$>$ Ensuring easy access to fresh drinking water for JDs.

$>$ Rewarding JDs achievements.

\section{Conclusion}

Bringing a group together to work on a number of areas important to JDs is starting to see some progress. Measuring the impact of these initiatives on JDs morale is challenging and we are exploring ways to do this effectively. In line with our people strategy at STHFT, we believe that staff who feel cared for will in return impart a higher level of care to their patients. 